\title{
STABILITY IN SPATIAL VOTING GAMES WITH RESTRICTED PREFERENCE MAXIMIZING
}

\author{
Thomas Bräuninger
}

\begin{abstract}
Spatial models of simple majority rule voting suggest that stable decisions are not likely to exist under normal circumstances. Yet this instability result stands in contrast to the stability observed in experiments. This article examines the effect of relaxing the assumption that voting is costless by requiring a proposal to be a finite distance closer to a member's ideal point than the pending proposal before it is regarded as attractive. Using the concept of the epsilon-core the article estimates the minimal decision costs that guarantee stable outcomes. It shows that the minimal costs are equal to the minimal finagle radius (Wuffle et al., 1989) and that the epsilon-core contains the finagle point which is close to the center of the yolk. While the analytical model establishes that the minimal costs are smaller than the yolk radius, computational simulations of majority voting by committees of size 3 to 101 suggest that this is a weak upper bound, only, as the ratio of minimal costs to the yolk radius usually is small and decreases as committee size approaches infinity.
\end{abstract}

KEY WORDS • decision costs $\bullet$ epsilon-core $\bullet$ stability $\bullet$ spatial voting models

\section{Introduction}

Majority-rule-based aggregation of preferences has largely been investigated in a setting referred to as spatial model of voting in which alternative policies are viewed as points in a convex space over which a finite set of voters have single-peaked preferences. Beginning with Hotelling's (1929) theory of economic competition, Black's (1958) theory of committee decision making, and Downs's (1957) economic theory of voting, spatial models have become common in the analysis of party competition, electoral behavior and legislative and government decision making. The most famous results, however, are discouraging with regard to democratic political processes: when alternatives are evaluated on two or more dimensions, Condorcet

Previous versions of this article were presented at the Annual Meeting of the Public Choice Society, San Diego, 2002, the Midwest Political Science Association National Conference, Chicago, 2003, and at the Max Planck Institute for Research into Economic Systems, Jena, 2004. Thanks to David Austen-Smith, Steven Callander and Macartan Humphreys for valuable advice, and to David Koehler for sharing his algorithm to compute the yolk. This work was supported by German Science Foundation grant \#BR1851/3-1/2. 
winners or undominated alternatives are unlikely to exist (Plott, 1967; Davis et al., 1972) and cycles of pairwise voting may be constructed that include any alternative (McKelvey, 1976; Schofield, 1978). Based on the empirical observation that there is more stability in real-world politics than theoretical models of social choice indicate (Tullock, 1981), scholars have studied the conditions that guarantee or favor the existence of stable, undominated outcomes. ${ }^{1}$ Following the initial focus on domain restrictions of preferences (Arrow, 1951) such as single-peakedness (Black, 1958), most of these studies concentrate on institutional arrangements such as super-majoritarian quotas (Greenberg, 1979), agenda setting and germaneness rules (Shepsle and Weingast, 1981), bi- or multi-cameralism (Hammond and Miller, 1987; Bräuninger, 2003) that lead to some sort of structure-induced equilibrium (Shepsle, 1979).

This article takes a different stance. It argues that even in the absence of dense institutional environments, there are numerous factors that may create majorityrule stability in a spatial context where it otherwise does not exist and that are related to individuals' ability or willingness to rank alternatives or vote on proposals rather than restrictive institutional rules. Examples of restricted preference maximization are the bias to vote for the incumbent, well-known in studies of voting behavior, or the restricted ability of committee members 'to make fine distinctions between alternatives without becoming indifferent' (McKelvey, 1976: 481). While the conventional preference-maximizing assumption has often been questioned, the conjecture that voting behavior that falls short of strictly distancebased preference maximizing is likely to lessen the effects of instability, has not been studied extensively (cf. Koehler, 2001).

In this article I contribute to this literature by considering what happens when relaxing the assumption that voting is costless for any committee member. More specifically, I model committee members as individuals that do not regard a proposal as attractive unless it is a finite distance closer to their ideal point than the pending proposal. The decisive question then is whether these thresholds or decision costs will induce majority-rule stability and if so, where stable outcomes are located in the policy space. Using the concept of the epsilon-core the article estimates the minimal decision costs that guarantee stable outcomes. It shows that the minimal costs are equal to the minimal finagle radius (Wuffle et al., 1989) and that the epsilon-core contains the finagle point which is close to the center of the yolk. While the analytical model establishes that the minimal costs are smaller than the yolk radius, computational simulations of majority voting by committees of size 3 to 101 suggest that this is a weak upper bound, only, as the ratio of minimal costs to the yolk radius usually is small and decreases as committee size approaches infinity. 


\section{Preference-induced Stability in Spatial Voting}

Indifference and alienation are two concepts that are used to analyze situations where agents abstain from voting so that stable outcomes become more likely. An agent may be indifferent when he perceives that there are only minor differences among the alternatives, whereas alienation refers to a situation where all options are outside the range of interesting, attractive alternatives (Hinich and Munger, 1997). Regarding voting behavior in mass elections, indifference and alienation have been incorporated in the Downsian spatial model (Riker and Ordeshook, 1968; Enelow and Hinich, 1984) but there are only a few studies that explore their impact on stability in situations of committee voting. Other conditions such as constraints on candidates' moves by previously publicly held positions (Samuelson, 1984; see also Laver, 2005) also seem to be more apt for electoral competition than committee voting.

Tovey (1991) discusses several assumptions concerning individual decision making that each lead to a modification of the conventional spatial model and that are likely to induce stability. These include bounded rationality (individuals have limited 'computational' abilities when making their choices), the presence of uncertainty as to the consequences of the proposal ('incumbency advantage'), or perceptual thresholds where individuals are not able to distinguish between proposals that are too similar. In the same vein, Feld and Grofman (1996) argue that voters may be neither willing to waste time considering alternatives that are only trivially different from one another nor to support large changes. They also provide analytical results on when restricted preference maximization under such 'noquibbling' and incrementalism norms leads to stable outcomes under majority rule.

Most recently, Koehler (2001) has readdressed the question of preference-induced stability in the context of committee voting. He considers thresholds or hurdles of minimal improvements that proposals must take to outweigh certain (though unspecified) decision or transaction costs: 'If $\varepsilon$ [the individual preference threshold] is large, members vote only for proposals that are substantial "improvements" on the status quo or the pending proposal' (p. 160; see also Tovey, 1991: 10). As a result, a proposal may attract an agent's vote only if it is to a certain extent closer to her ideal point than the pending proposal. Decision-making outcomes are thus more likely to be stable because any further decision requires (transaction) cost-offsetting improvements on the side of a majority of committee members. This setting leads to the concept of the quasi- or epsilon-core developed by Shapley and Shubik (1966) in their study of pure exchange economies with participating traders having non-convex preferences. In these markets the core is often vacuous, and in order to 'resolve the inherent instability of this situation, we must resort to social, cultural, or institutional restraints' (p. 823). They consider $\varepsilon$ as an organizational cost prerequisite to cooperative action, and quasi-cores as a way of taking into account the costs of coalition formation, for example. While the epsilon-core has become an established concept in the study of general $n$-person games with coalition structure (Maschler et al., 
1979; Nakayama and Naitoh, 1999; Kovalenkov and Wooders, 2000) it has seldom been applied to spatial models of voting.

One exception is Salant and Goodstein (1990). Studying committee behavior in majority rule voting experiments, they consider threshold effects in order to explain why their experimental committees frequently failed to choose the Condorcet alternative. They estimate the minimal improvements that players might consider 'not worth the trouble' (p. 302). Empirically they find that even for pretty small thresholds the predictive power of the modified spatial model is impressive. What is more, when applied to the experimental results of independent studies (Fiorina and Plott, 1978; Eavey and Miller, 1984), 95 per cent of all committee choices are found in the selection set of undominated points. A second exception is Herzberg and Wilson (1991) who subject a spatial voting model incorporating a form of transaction costs to empirical testing using laboratory experimental data. They find that while the evidence for predictions of outcomes under the modified model are not overly compelling, the experiments 'clearly point to the salience of agenda costs for the collective choice process' (Herzberg and Wilson, 1991: 197). A third exception is Tovey (1991), who argues that the epsilon-core may reflect the presence of costs of change but may also be justified by the limited computational abilities of agents in a bounded rationality framework (see also Sloss, 1973). He shows that for the class of centered distributions of ideal points the epsilon-core generically exists for any $\varepsilon>0$ as the number of ideal points goes to infinity (Tovey, 1991: 11). For non-centered distributions, that is when the yolk radius is strictly larger then zero, the yolk center proves to be an undominated point if the threshold $\varepsilon$ is strictly larger than the yolk radius (Tovey, 1991: 20). The simulation study of Koehler (2001) supports these earlier experimental results and theoretical accounts indicating that 'stability can be achieved at relatively low individual preference thresholds' and stable outcomes 'are found near the [yolk] center of the preference space' (p. 166).

Given these theoretical results on finite committees drawn randomly out of (continuous) probability distributions and the simulation and experimental findings on small committees, the analytical questions that remain are, when does the epsilon-core in committees of finite size exist, where is it located and how large is it? In the following, some implications of the individual preference threshold setting for the existence of majority rule equilibrium are studied. In particular, I show that as the preference threshold increases, the first stable point that emerges is the so-called finagle point, not the yolk center.

\section{Committee Voting with Decision Costs}

This section introduces a simple decision or transaction cost term to the conventional spatial model of voting. Based on modified agents' preferences the epsiloncore is defined. The epsilon-core is a superset of the conventional core and may 
exist when the latter is empty. It is argued that the yolk radius is an upper bound for the minimal decision costs that guarantee that stable outcomes exist. In this case the center of the yolk is an element of the epsilon-core.

Suppose that the policy space is given by a nonempty, compact and strictly convex subset of the multi-dimensional Euclidean space, $X \subseteq \mathfrak{R}^{k}$. The set of agents involved in decision-making within the committee is given by $N=\{1, \ldots, n\}$, $n$ odd. Decisions are taken by simple majority. In the conventional spatial model, agents are assumed to have strictly convex, single-peaked preferences over the set of alternatives. This means that for any agent a so-called ideal point $x_{i}$ exists that represents its most preferred policy and any other alternative is ranked lower relative to its - probably weighted - distance from the ideal point. More specifically, assume that all ideal points $x_{i}$ are unique and agents have symmetric Euclidean preferences so that an alternative $x$ is strictly preferred to alternative $y, x P_{i} y$, if and only if $\left\|x_{i}-x\right\|<\left\|x_{i}-y\right\|$. It is weakly preferred to $y, x R_{i} y$, if and only if $\left\|x_{i}-x\right\| \leq\left\|x_{i}-y\right\|$.

In the conventional model, agents would vote for policy change if and only if the new policy is more beneficial than the status quo. The act of voting itself is assumed to be costless. Casting one's vote, however, requires the acquisition of information, evaluation of competing policies, deliberation and, at least, time. Introducing such decision or transaction costs to the spatial model means that the utility of a policy change must outweigh the costs that are incurred in the process of decision-making itself. Let these costs or voting threshold be denoted by $\varepsilon$. Throughout this article, I assume that they are not agent-specific and independent from the decision to be made. ${ }^{2}$ Then agent $i$ (strictly) prefers an alternative $x$ to alternative $y, x P_{i}^{\varepsilon} y$, if and only if $\left\|x_{i}-x\right\|<\left\|x_{i}-y\right\|-\varepsilon$; the agent weakly prefers $x$ to $y, x R_{i}^{\varepsilon} y$, if and only if $\left\|x_{\mathrm{i}}-x\right\| \leq\left\|x_{i}-y\right\|+\varepsilon$. Note that for any $\varepsilon>0$, the binary relation $R_{i}^{\varepsilon}$ is complete, reflexive, and quasi-transitive, but not transitive. The following definition presents the standard terminology of the preferred set, the winset, and the core and extends it to $\varepsilon$-based preference relations.

Definition 1. (i) The preferred set of $x$ (regarding the binary relations $P_{i}$ and $P_{i}^{\varepsilon}$, respectively) contains all policies that agent $i$ strictly prefers to policy $x$ :

$$
P_{i}(x)=\left\{y \in X: y P_{i} x\right\} \text { and } P_{i}^{\varepsilon}(x)=\left\{y \in X: y P_{i}^{\varepsilon} x\right\} .
$$

(ii) Let $\boldsymbol{W}$ denote the set of winning coalitions, that is, the sets of agents that the voting rules empower to change the status quo. Say that some policy $y$ is socially

2. It might be argued that devising a proposal incrementally better for a majority of voters is much more costly for the agenda setter than figuring out for a particular committee member what he or she prefers. This would result in a model with an (additional) restriction on the preference maximizing of the agenda setter. In this article, I refrain from considering these proposal costs. For a different setup where $\varepsilon$ is an agent-specific decision cost and $x$ has to be at least a certain percentage closer to the individual's ideal points than $y$, for example $x$ is preferred to $y$ if and only if $\left\|x_{i}-x\right\|<\left(1-\varepsilon_{i}\right)$ $\left\|x_{i}-y\right\|$, see Sloss (1973), and Ganghof and Bräuninger (2006). 
preferred to policy $x$ if there is a winning coalition, $S \in \boldsymbol{W}$, whose members all prefer $y$ to $x$. The winset of $x$ then is the set of all policies that are socially preferred to $x$ :

$$
\begin{aligned}
W(x) & =\left\{y \in X: \exists S \in W \text { so that } y \in P_{i}(x) \text { for any } i \in S\right\} \text { and } \\
W^{\varepsilon}(x) & =\left\{y \in X: \exists S \in W \text { so that } y \in P_{i}^{\varepsilon}(x) \text { for any } i \in S\right\} .
\end{aligned}
$$

(iii) The core is the set of all alternatives that cannot be upset by any other alternative:

$$
C=\{x \in X: W(x)=\varnothing\} \text { and } C^{\varepsilon}=\left\{x \in X: W^{\varepsilon}(x)=\varnothing\right\} .
$$

I shall illustrate these definitions in two dimensions. For example, Figure 1 shows a set of ideal points for three committee members, their preferred set of a pending proposal $x$ for both conventional and $\varepsilon$-based preferences relations. For any ideal point $x_{i}$ and pending proposal $x$, the $\varepsilon$-preferred set of $x, P_{i}^{\varepsilon}(x)$, is a subset of the conventional preferred set, $P_{i}(x)$, and, accordingly, $W^{\varepsilon}(x)$ is contained in the conventional winset, $W(x)$. For large values of $\varepsilon$ the $\varepsilon$-winset of $x$ will be empty so that $x$ is in the epsilon-core though the conventional core is empty.

Denote the open ball with center $x$ and radius $\varepsilon$ by $D(x, \varepsilon)=\left\{y \in \mathfrak{R}^{k}: \| y-x\right.$ $\|<\varepsilon\}$. The following is then straightforward.

Lemma 1 . For any $i \in N, x \in X$ :

(i) $P_{i}^{\varepsilon}(x) \subseteq P_{i}(x), W^{\varepsilon}(x) \subseteq W(x)$, and $C \subseteq C^{\varepsilon}$.

(ii) $D(x, \varepsilon) \cap W^{\varepsilon}(x)=\varnothing$ for any $\varepsilon>0$.

(iii) Let $\delta>0$ so that $W(x) \subseteq D(x, \delta)$. If $\varepsilon>\delta$ then $x \in C^{\varepsilon}$.

\section{Proof:}

(i) is immediate from Definition 1.

(ii) For any $y \in D(x, \varepsilon)$, we know that $\left|\left\|y-x_{i}\right\|-\left\|x-x_{i}\right\|\right| \leq \varepsilon$ for any agent $i$. Thus $y \notin P_{i}^{\varepsilon}(x)$ and $y \notin W^{\varepsilon}(x)$. The open ball $D(x, \varepsilon)$ is therefore an inner hole of $W^{\varepsilon}(x)$.

(iii) We have to show that there is no policy $y$ so that $y \in W^{\varepsilon}(x)$. First, assume that $y \notin D(x, \delta)$ which implies $y \notin W(x)$. Since $W^{\varepsilon}(x) \subseteq W(x), y$ is not socially preferred to $x$. Second, let $y \in D(x, \delta)$. Then $\left|\left\|y-x_{i}\right\|-\left\|x-x_{i}\right\|\right| \leq \delta<\varepsilon$ for any i. Thus, again, $y \notin W^{\varepsilon}(x)$.

This means that the $\varepsilon$-winset is contained in the conventional winset and the open ball $D(x, \varepsilon)$ represents an inner hole of $W^{\varepsilon}(x)$. Note that in contrast to the conventional winset $W(x)$, the $\varepsilon$-winset $W^{\varepsilon}(x)$ is not starlike and not connected.

Given the fundamental importance of stable decisions it is natural to ask, under which conditions the epsilon-core is not empty. To this end, I first introduce the concept of the yolk which is the smallest circle that intersects all 


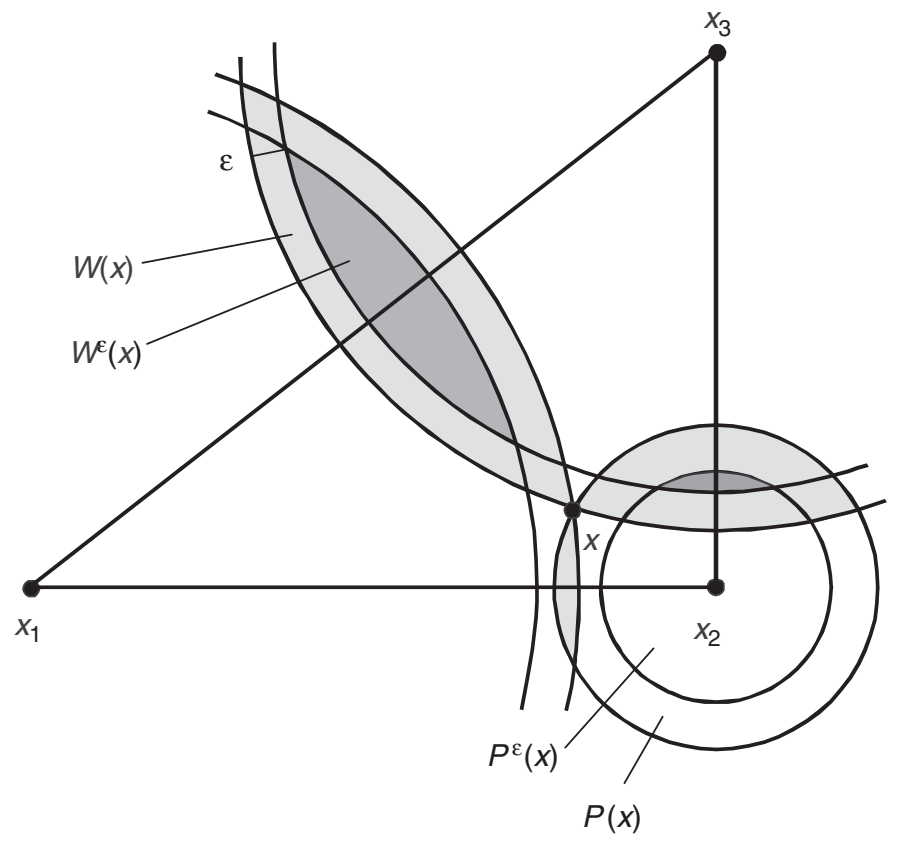

Figure 1. Preferred Sets and Winsets for a Three-member Committee

median hyperplanes (Ferejohn et al., 1984; McKelvey, 1976). The yolk radius can be considered as a measure for the extent to which the distribution of ideal points 'deviates from one that would generate majority-rule equilibrium' (Miller et al., 1989: 405). From the yolk, lower and upper bounds of any point's winset can be derived. Let $c$ be the center and $r$ be the radius of the yolk, and denote the distance between an arbitrary point $x$ and the center of the yolk by $d$. Ferejohn et al. (1984) show that upper and lower bounds of the winset $W(x)$ are given by two circles around $c$ with radii $d-2 r$ and $d+2 r$, i.e. $D(c, d-2 r) \subseteq W(x) \subseteq$ $D(c, d+2 r) .^{3}$ The next lemma states that there is a similar upper bound for the threshold-based winset:

Lemma 2. For any $x \in X$ and $\varepsilon>0$ :

$$
\mathrm{W}^{\varepsilon}(x) \subseteq D(c, d+2 r-\varepsilon) \backslash D(x, \varepsilon),
$$

where $c$ is the center, $r$ is the radius of the yolk and $d$ is the distance of $c$ and $x$.

3. More specific bounds can be defined in terms of cardioids with center $c$, cusps at $x$, radii $d$ and eccentricities of $2 r$ and $-2 r$. 
Proof: (i) I first show that for any alternative in the $\varepsilon$-winset of $x, y \in W^{\varepsilon}(x)$, there is an alternative in the conventional winset, $z \in W(x)$, so that $\|\mathrm{c}-y\|<\|\mathrm{c}-z\|-\varepsilon$. To this end let

$$
z=y+\frac{\varepsilon}{\|y-c\|}(y-c)
$$

and recall that as $y \in W^{\varepsilon}(x)$ there is an agent set $S$ so that $\left\|x_{i}-y\right\|<\left\|x_{i}-x\right\|-\varepsilon$ for any $i \in \mathrm{S}$. Then, by the triangle inequality, we have that for any $i \in \mathrm{S}$ :

$$
\left\|x_{i}-z\right\|=\left\|x_{i}-y-\frac{\varepsilon}{\|y-c\|}(y-c)\right\| \leq\left\|x_{i}-y\right\|+\varepsilon<\left\|x_{i}-x\right\|
$$

It follows that $z \in W(x)$.

(ii) From (i) follows that $W(x)$ is in a distance of at least $\varepsilon$ from the most distant point of $W^{\varepsilon}(x)$ so that $W^{\varepsilon}(x) \subseteq D(c, d+2 r-\varepsilon)$. Combining this with Lemma 1(ii) we have what was claimed, i.e. $W^{\varepsilon}(x) \subseteq D(c, d+2 r-\varepsilon) \backslash \mathrm{D}(x, \varepsilon)$.

Figure 2 illustrates the 'donut' shape of the boundary set that contains $W^{\varepsilon}(x)$ for the example of a committee with three agents with ideal points in a two-dimensional space. While the circle centered at $x$ with radius $d+2 r-\varepsilon$ represents the upper bound of the $\varepsilon$-winset, no points in an $\varepsilon$-environment of $x$ can be altered and are thus not part of the $\varepsilon$-winset. From Lemma 2 follows:

Proposition 1. Let $c$ be the center and $r$ be the radius of the yolk. If $\varepsilon>r$ then $c \in C^{\varepsilon}$.

Proof: Consider the case $x=c$. According to Lemma 2, $W^{\varepsilon}(c) \subseteq D(c, 2 r-\varepsilon) \backslash D(c, \varepsilon)$ so that $W^{\varepsilon}(c)=\varnothing$ if $\varepsilon>r$.

In words, Proposition 1 states that if the costs associated with the act of voting are sufficiently larger than zero, the epsilon-core of the spatial voting game will be not empty. More specifically if $\varepsilon$ is larger than the radius of the yolk, the yolk center will be an element of the epsilon-core. This is a noteworthy result as the yolk generally shrinks as the number of voters increases in large committees or in mass elections with a large number of voters (Koehler, 1990; Tovey, 1991; Hug, 1999; Godfrey and Miller, 2005). In this case, the center of the yolk is an element of the epsilon-core even for rather small voting thresholds. Moreover, for the special case of a random sample of ideal points drawn from a centered (e.g. uniform or normal) distribution, the epsilon-core exists with probability 1 for any $\varepsilon>0$ as the number of ideal points goes to infinity (Tovey, 1991). Consequently, for large electorates the center of the yolk is a likely stable outcome. The following section shows, however, that a different point in the space is an even better approximation: the finagle point. 


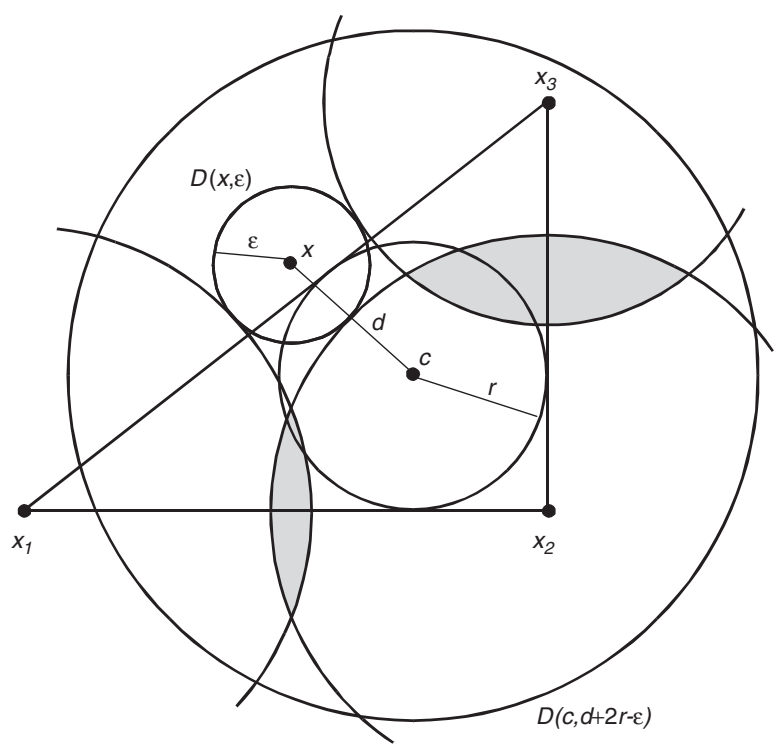

Figure 2. Donut Shape of Boundary of $\varepsilon$-winset for a Three-member Committee

\section{Minimal Voting Threshold and the Finagle Point}

Given the stability result for sufficient large voting thresholds, a subsequent question is: what are the minimal thresholds that guarantee the existence of stable outcomes? This section shows that the minimal decision cost or threshold for the epsilon-core not to be empty is equal to the minimal finagle radius. In this case the finagle point is an element of the corresponding epsilon-core.

The finagle point is the point in the policy space with a minimal finagle radius: 'from it a candidate can, with only minuscule changes in his initial policy location, find a response to any challenger that will defeat that challenger' (Wuffle et al., 1989: 348). The finagle point is close to the center of the yolk but in general does not coincide with it. More formally, the following definitions are used (Wuffle et al., 1989: 350):

Definition 2. (i) A set of points $V$ is a V-Mess for a majority voting game if for any $x \notin V$ there is a $y \in V$ so that $y \in W(x)$, i.e. it is externally stable.

(ii) The point that minimizes the distance to the maximally distant point in $V$ is the center of $V$, the maximum distance is the radius of $V$.

(iii) The finagle radius of a point is the radius of the minimal circular V-Mess centered around it. The point with minimal finagle radius is the finagle point.

(iv) The minimal voting threshold $\varepsilon^{*}$ is the minimal voting threshold $\varepsilon \geq 0$ so that $\mathrm{C}^{\varepsilon} \neq \varnothing$. 
Proposition 2. Let $f$ be the finagle point, $r_{f}$ the minimal finagle radius, and $\varepsilon^{*}$ the minimal voting threshold for a majority voting game. Then:

$$
\varepsilon^{*}=r_{f} \quad \text { and } \quad f \in C^{\varepsilon^{*}} .
$$

Proof: (i) If the conventional core is empty, $C \neq \varnothing$, then $\varepsilon^{*}=0$. But since the number of agents, $n$, is odd, any $x \in \mathrm{C}$ is a V-Mess so that $r_{f}=0$.

(ii) In general, however, $C=\varnothing$. In this case we have $\varepsilon^{*}>0$, but there is also an upper bound for $\varepsilon$ because for sufficiently large thresholds, there is always some alternative that is stable. I now show that for any circular V-Mess, its center is an element of the $\varepsilon$-core if $\varepsilon$ equals its radius (or is larger). Formally, let $D\left(x, r_{x}\right)$ be the open disk with center $x$ and radius $r_{x}$, let $\overline{D\left(x, r_{x}\right)}$ be its closure and let

$$
V=\left\{\overline{D\left(x, r_{x}\right)}: x \in \mathfrak{R}^{k}, r_{x}>0, \overline{D\left(x, r_{x}\right)} \text { is V-Mess }\right\}
$$

denote the set of all circular V-Messes. We wish to show that

$$
x \in \mathrm{C}^{r} \text { for any } \overline{D\left(x, r_{x}\right)} \in V .
$$

To see this, we have to show that for any $\overline{D\left(x, r_{x}\right)}$, the winset $W^{r_{x}}(x)$ is empty, i.e. there is no alternative $y \in W^{r} x(x)$. By way of contradiction, suppose that there is a $y \in X$ with $y \in W^{r} x(x)$. There are two cases:

Case 1: $y \in \overline{D\left(x, r_{x}\right)}$. Then $\|y-x\| \leq \varepsilon$ thus $y \notin W^{r} x(x)$. Hence we have a contradiction. Case 2: $y \notin \overline{D\left(x, r_{x}\right)}$. Then, as $\overline{D\left(x, r_{x}\right)}$ is a V-Mess, there is at least one alternative that dominates $y$, i.e. there is a $\chi \in X$ with

$$
\|x-\chi\| \leq r_{x}
$$

and a winning coalition $S \in W$ so that for any coalition agent's ideal point $x_{i}$ :

$$
\left\|x^{i}-\chi\right\|<\left\|x^{i}-y\right\| \text {. }
$$

For the triangles $x \chi x^{i}$ we have:

$$
\|\| x_{i}-\chi\|-\| x_{i}-x\|\mid<\| \chi-x \| .
$$

Combining (a) to (c), we have $\left\|x_{i}-y\right\|-\left\|x_{i}-x\right\|>\left\|x_{i}-\chi\right\|-\left\|x_{i}-x\right\|>-$ $\|x-\chi\| \geq-r_{x}$. In words, this means that alternative $x$ is weakly preferred to alternative $y$ which implies that $y$ cannot be element of the winset of $x$, i.e. $y \notin W^{r} x(x)$. This, however, is a contradiction. ${ }^{4}$

Proposition 2 provides an alternative proof of Proposition 1 as the radius of the yolk is an upper bound for the minimal finagle radius (Wuffle et al., 1989). For

4. Note that if $C^{\varepsilon}$ is strictly convex for any $\varepsilon>0$, then one can show that the epsilon-core coincides with the finagle point, i.e. $\{f\}=C^{\varepsilon^{*}}$. Otherwise one could always find an open ball with center $z$ in the interior of the $\varepsilon$-core and a $\delta$ such that $z \in(\varepsilon-\delta)$-core. Convexity of $C^{\varepsilon}$, however, is not straightforward as the Pareto set of an arbitrary winning coalition generally is not convex. 
most configurations, however, the minimal finagle radius is much smaller than the yolk radius. Figure 3 illustrates Proposition 2 with respect to the three-voter case. While the yolk is tangent to any side of the triangle, the minimal finagle radius is the radius of the inner circle $\left(\varepsilon^{*}\right)$ that is tangent to all three outer circles. The outer circles are centered at agents' ideal points and pass through those points on the triangle where the yolk is tangent. The minimal finagle radius represents the smallest decision costs or voting threshold where a majority-rule equilibrium exists. In this case, the finagle point $(f)$ rather than the yolk center $(c)$ is a stable outcome.

For the three-actor case, the minimal finagle radius is always smaller than the yolk radius. With three actors and ideal points $x 1, x 2$ and $x 3$, the minimal finagle radius is given by:

$$
\begin{aligned}
r_{f} & =\frac{1}{\alpha+\beta+\gamma+2 \sqrt{\alpha \beta+\beta \gamma+\alpha \gamma}}, \text { where } \alpha=\frac{1}{p-\left\|x_{2}-x_{3}\right\|}, \\
\beta & =\frac{1}{p-\left\|x_{1}-x_{3}\right\|}, \\
\gamma & =\frac{1}{p-\left\|x_{1}-x_{2}\right\|}, \text { and } p=\frac{\left\|x_{1}-x_{2}\right\|+\left\|x_{2}-x_{3}\right\|+\left\|x_{1}-x_{3}\right\|}{2} .
\end{aligned}
$$

As a result, the ratio of the minimal finagle radius and that of the yolk is at most $r_{f} / r=(2+\sqrt{3})^{-1} \approx 0.267$ (Wuffle et al. 1989: 362). Is this also an upper bound for, at least generically, all ideal point configurations in the $n$-actor case? The following section shows that while this is not the case, the average ratio of minimal threshold to yolk radius in fact is considerably smaller than .267 and simulation results suggest that it is decreasing with the number of actors.

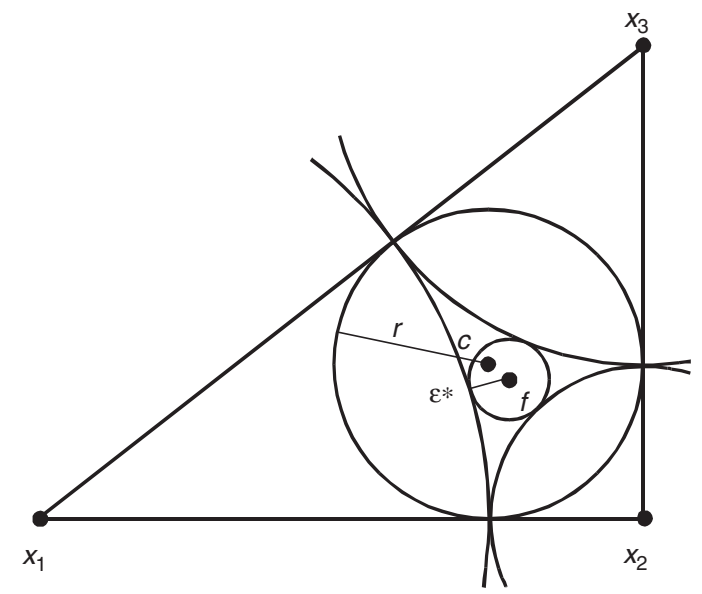

Figure 3. Minimal Threshold $\varepsilon^{*}$ for a Three-member Committee 


\section{Estimating the Minimal Threshold}

Analytical methods would be preferable for estimating the threshold $\varepsilon^{*}$ above which stable outcomes under simple majority rule exist for a specific configuration of ideal points. While the question can easily be answered for the three-actor case, geometric or algebraic procedures are cumbersome even for five-actor committees and become increasingly awkward for larger committees. Here simulation results can extend analytic conclusions as they estimate empirical parameters rather than analytical bounds, in particular when we are interested in questions of the type, 'How likely are stable outcomes in typical committees?' instead of investigating whether there exist rare, non-generic cases where the parameter in question is exceptionally large (or small).

To analyze the present setting, in which proposals are required to be at least a finite distance closer to a committee member's ideal point to gain his vote, a computational model was built. To this end, first the number of ideal points $n$ was selected randomly from a uniform distribution over the set of odd numbers in the range $3 \leq n \leq 101$. Second, $n$ ideal points were selected from a uniform distribution over a two-dimensional 'policy space' $X=[0,10] \times[0,10] .{ }^{5}$ Given such a committee situation, an upper bound for the minimal epsilon that guarantees a stable outcome and the spatial location of these outcomes were estimated. ${ }^{6}$ This procedure was carried out 4000 times generating 4000 ideal point configurations and estimates of the threshold $\varepsilon^{*}$ and the stable outcome $o^{*}$. The results of this large number of experiments are reported in the subsequent tables and figures.

The algorithm used to estimate $\varepsilon^{*}$ and $o^{*}$ calculates an approximation $C(\varepsilon)$ of the epsilon-core for a sufficient large $\varepsilon$ and then decreases $\varepsilon$ gradually until the area of $C(\varepsilon)$ is reasonably small so that $\varepsilon$ is an approximation for $\varepsilon^{*}{ }^{7}$ The algorithm proceeds in three steps:

- Step 1: Use two times the yolk radius as the initial value for the voting threshold $(\varepsilon=2 r)$ and the yolk center as the initial value for the center of the $\varepsilon$-core $(z=c)$. We know from Proposition 2 that for $\varepsilon=r, c \in C(\varepsilon)$. As we do not

5. The simulation is limited to two dimensions for three reasons. First, most analyses of spatial models of committee voting are limited to two dimensions, thus sticking to the two-dimensional case will allow for direct comparison to previous studies (Wuffle et al., 1989; Hug, 1999; Koehler, 2001). Second, two-dimensional results usually apply to higher dimensions (Miller et al., 1989; Koehler, 1996). Third, a huge body of studies of a variety of political systems has demonstrated persuasively that most legislative decisions can be represented and explained on two dimensions (e.g. Poole and Rosenthal, 1997). Finally, the choice of a uniform rather than normal random distribution of ideal points is also for the sake of comparability with the study of Koehler (2001). I outline results for a bivariate normal distribution in the text.

6. Note that if $C^{\varepsilon}$ is strictly convex, $C^{\varepsilon^{*}}$ will contain exactly one point so that the estimated upper bound for $\varepsilon^{*}$ is also an approximation for $\varepsilon^{*}$.

7. Yolks and $\varepsilon^{*}$-cores are calculated using the program Mineps (available at http://www.tbraeuninger.de/download.html) which requires the commercial program Gauss. 
know whether or not $r$ is the least upper bound of $\varepsilon^{*}$ (so that the interior of $C(r)$ is empty) we use two times the yolk radius to ensure that the initial $C(r)$ has a non-empty interior.

- Step 2: Approximate location and size of $C(\varepsilon)$ by finding the boundary points of $C(\varepsilon)$ on any ray from $z$ specified by angle $\theta=2 \pi k / 16$ for $k=1, \ldots, 16$. The resulting $16 n$-polygon is a subset of $C(\varepsilon)$ with area $A$, and center of gravity $c_{P}$. If $A<\pi r^{2} / 10^{10}$, set $\varepsilon^{*}=\varepsilon$ and $o^{*}=c_{P}{ }^{8}$ Otherwise proceed with step 3 .

- Step 3: Denote by $r_{P}$ the radius of the largest circle with center $c_{P}$ that can be inscribed in the $16 n$-polygon. The new threshold $\varepsilon$ then is $\varepsilon-r_{P} / 2^{m}$ (where $m$ is chosen so that $W\left(c_{P}\right)$ is empty), the new approximate center of the $\varepsilon$-core is $z=c_{P}$. Proceed with step 2 .

The upper part of Table 1 shows the distribution of committee sizes and members' ideal points for the 4000 experiments that were run. The figures do not deviate from what we would expect to see. The two-dimensional mean of committee members' ideal points is, on average, close to the center of the space at $(5 ; 5)$ with an average two-dimensional variance of 16.6 as expected for a uniform

Table 1. Summary Statistics of 4000 Experiments

\begin{tabular}{|c|c|c|c|c|}
\hline & Mean & SD & Min & $\operatorname{Max}$ \\
\hline Number of actors & 52.0075 & 28.8449 & 3 & 101 \\
\hline \multicolumn{5}{|l|}{ Mean of ideal point position } \\
\hline$x$ coordinate & 5.0017 & .5663 & 1.2613 & 9.6488 \\
\hline$y$ coordinate & 4.9876 & .5852 & .9688 & 9.4697 \\
\hline \multicolumn{5}{|l|}{ Standard deviation of ideal point position } \\
\hline$x$ coordinate & 2.8705 & .3099 & 1498 & 4.8152 \\
\hline$y$ coordinate & 2.8636 & .3230 & .2173 & 5.1182 \\
\hline Two-dimensional variance & 16.6404 & 2.4546 & 1.6576 & 40.6692 \\
\hline Yolk radius $(r)$ & .5807 & .2482 & .0076 & 2.3956 \\
\hline \multicolumn{5}{|l|}{ Yolk center } \\
\hline$x$ coordinate & 4.9958 & .7501 & 9696 & 9.6776 \\
\hline$y$ coordinate & 4.9815 & .7554 & .8317 & 9.5769 \\
\hline Estimate of minimal threshold $\left(\varepsilon^{*}\right)$ & .0755 & .0671 & .0002 & 6337 \\
\hline \multicolumn{5}{|l|}{ Approximate location of $\varepsilon^{*}$-core } \\
\hline$x$ coordinate & 4.9948 & .7680 & .8913 & 9.7816 \\
\hline$y$ coordinate & 4.9813 & .7694 & .5956 & 9.4043 \\
\hline Distance yolk center-to- $\mathcal{E}^{*}$-core & .1193 & .0815 & .0009 & .6141 \\
\hline $\begin{array}{l}\text { Ratio of distance yolk center-to- } \varepsilon^{*} \text {-core } \\
\text { to yolk radius }\end{array}$ & .2162 & .1389 & .0015 & 0.9630 \\
\hline Ratio of $\varepsilon^{*}$ to yolk radius & .1159 & .0471 & .02356 & .3523 \\
\hline
\end{tabular}

$N=4000$ experiments; number of actors drawn from a uniform distribution over the set of odd numbers between 3 and 101; ideal points drawn from a uniform distribution over the square $[0,10] \times[0,10]$.

8. This means that the algorithm stops if the approximate area of the $\varepsilon$-core is $10^{10}$ times smaller than the area of the yolk. 
distribution of ideal points. ${ }^{9}$ Results on the spatial location of the yolk and the $\varepsilon^{*}$-core are shown in the lower part of Table 1 . Yolk centers and approximate $\varepsilon^{*}$-cores cluster in the middle of the policy space as expected (with means of about 4.99 and standard deviations of .76 on each of the two dimensions). While the average yolk radius is considerable (.5807), the simulation results reveal that the minimal threshold that guarantees the existence of a stable outcome is on average much smaller, ranging from .0002 to .63 with a mean threshold size of .0755. More specifically, the $\varepsilon^{*}$-to-yolk radius ratio is small with an average ratio of .1159 and a maximum ratio of .3523 . This is in line with Proposition 1 stating that the $\varepsilon$-core is non-empty for $\varepsilon>r$ but it also suggests that the smallest upper bound of the ratio for the three-actor case which is $r_{f} / r \approx 0.267$ is not an upper bound for the $n$-actor case.

Figure 4 shows the location of the yolk center and the $\varepsilon^{*}$-core for the first 100 experiments that were run. Outcomes are located almost anywhere in the

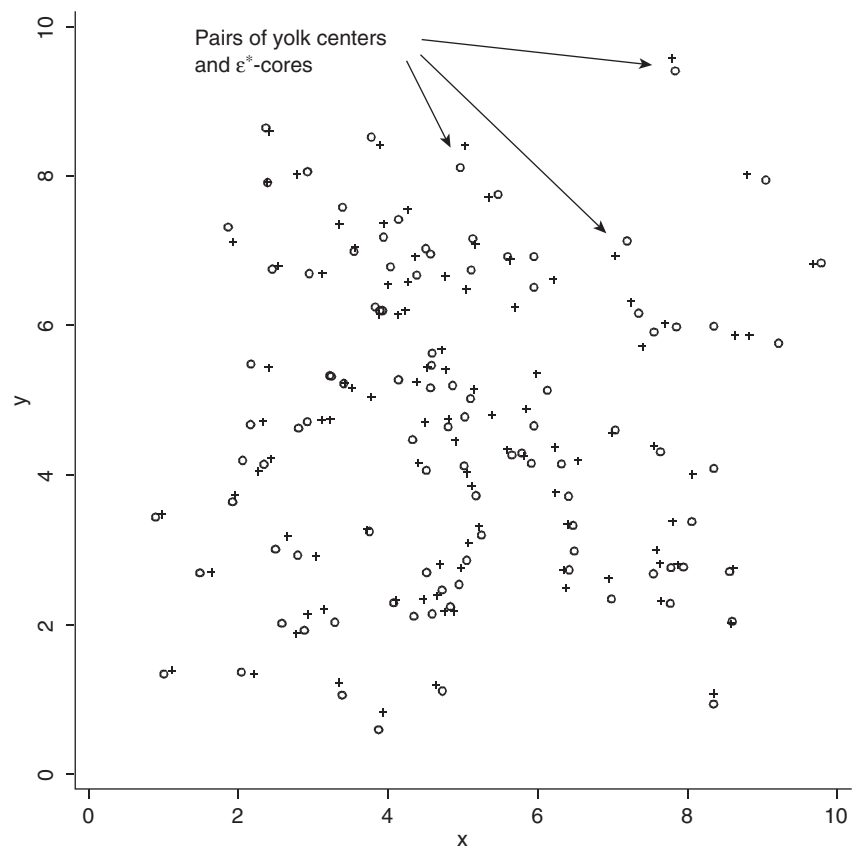

Figure 4. Pairing-up of Yolk Centers (+) and $\varepsilon^{*}$-cores (o) for 100 Experiments

9. For a uniform distribution over the 10-by-10 square the expected two-dimensional variance is $V=10^{2}(n-1) / 6 n$, where $n$ is the number of ideal points (Koehler 1996: 161). Thus for committee sizes $n=3, \ldots, 101$ drawn from a uniform distribution the expected variance is $\sum_{n=1}^{50} \frac{100.2 n}{6(2 n+1)}=16.02$. 
two-dimensional alternative space but the yolk center and $\varepsilon^{*}$-core are always located in a small distance to each other. Yet, they never coincide. ${ }^{10}$ Moreover, Table 1 shows that the distance is always smaller than the yolk radius suggesting that the $\varepsilon^{*}$-core or finagle point is an element of the yolk (Wuffle et al., 1989).

Figures 5-7 present three panels reporting the yolk radius, the estimated minimal voting threshold and the minimal threshold-to-yolk radius ratio by committee size. The first panel replicates findings from previous analyses of the yolk showing that the yolk radius on average decreases as the size of the committee increases. The same is true for the variance in the yolk radius for a given committee size. This is consistent with previous simulation results, for example those reported in Koehler (1990) and Hug (1999). The second panel shows the magnitude of the minimal threshold by committee size. For a three-actor committee, decision costs of an average of no more than .2 are sufficient to guarantee stable outcomes under simple majority rule. With 23 agents, the average minimal threshold decreases to .1, with a 101-agent committee the average

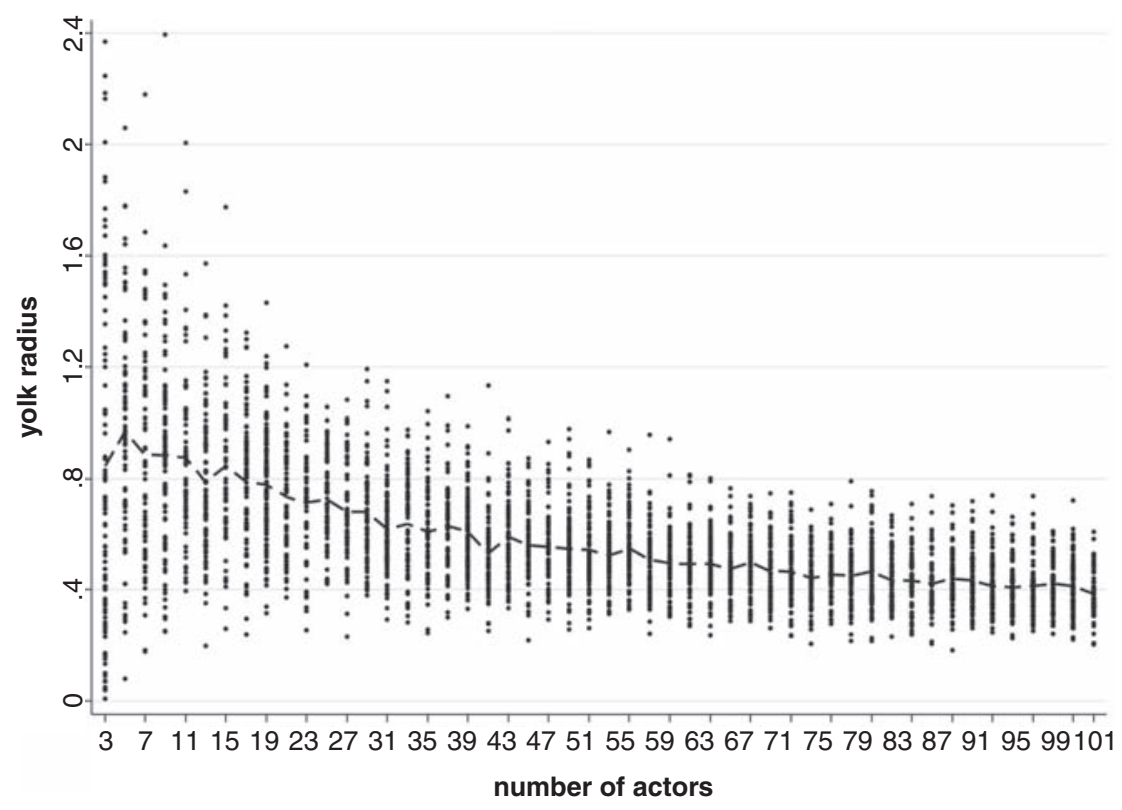

Note. The solid line is the average yolk radius.

Figure 5. Yolk Radius by Committee Size

10. Yolk center and $\varepsilon^{*}$-core coincide, for instance, when the ideal points of three agents form an equilateral triangle, and generally, for any symmetric ideal point configuration. 


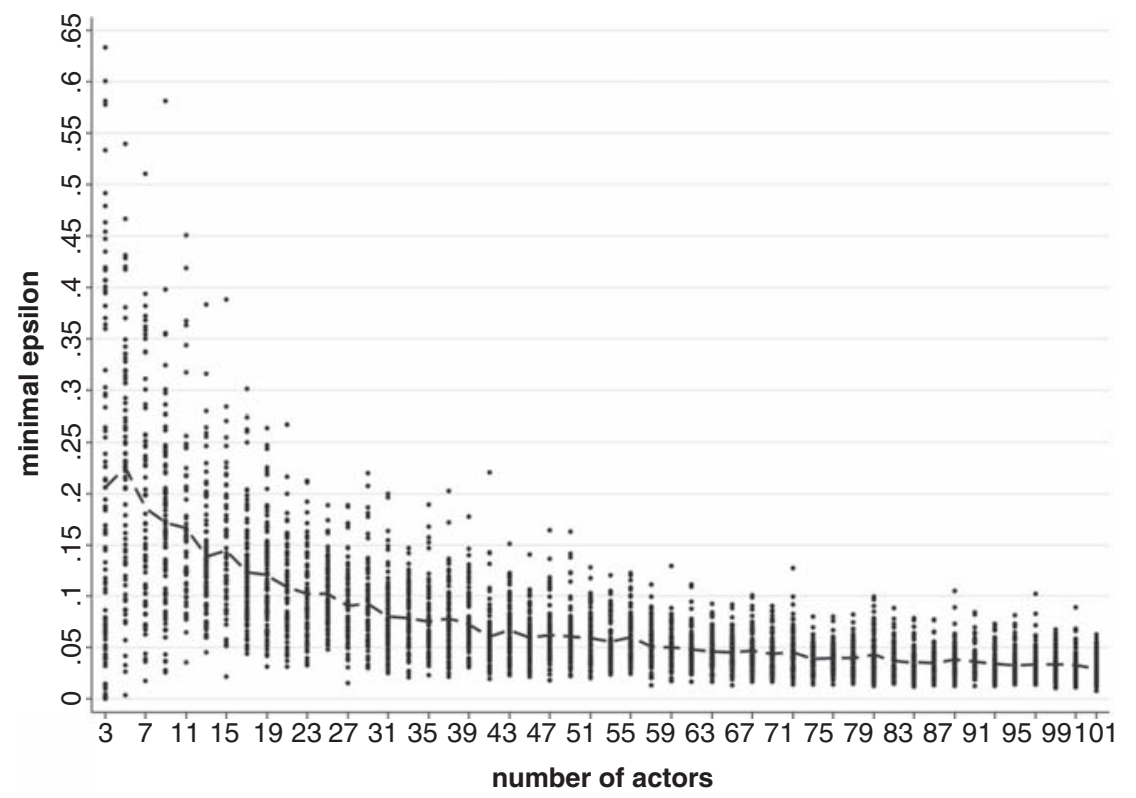

Note. The solid line is the average minimal threshold

Figure 6. Minimal Threshold by Committee Size

threshold is .03. The third panel presents the key finding showing the minimal threshold-to-yolk radius ratio by committee size. Three findings are noteworthy: first, 'confirming' the analytical findings, the minimal threshold for a three-agent committee is at most .2679. Second, both the average ratio and the variance in the ratio decreases with the committee size. Third, at least for small committee sizes $(3<n \leq 15)$ there are ideal point configurations where $\varepsilon^{*}$ exceeds the three-actor-boundary of .2679. Moreover, despite the fact that the ratio is decreasing for large $n$, the average $\varepsilon^{*}$ for the five-actor committee is larger than that for the three-actor committee. Finally, replicating the analysis using a bivariate normal distribution to draw actors' ideal points, yields similar results. In a sample of 4000 experiments, the $\varepsilon^{*}$-to-yolk radius ratio has an average of .1164, the mean ratio between the yolk center-to- $\mathcal{E}^{*}$-core distance and the yolk radius is .2344.

This all is consistent with Koehler's (2001) simulation results. In his setting, stable outcomes are generated when, on average, $\varepsilon / r=0.279$ and $n=72.733$, while simulations were run for odd-sized committees $(21 \leq n \leq 101)$ with ideal points selected randomly from a uniform distribution over a two-dimensional square. Supplementing these findings, the present study shows that the actual average minimal threshold $\varepsilon^{*}$ is much smaller. 


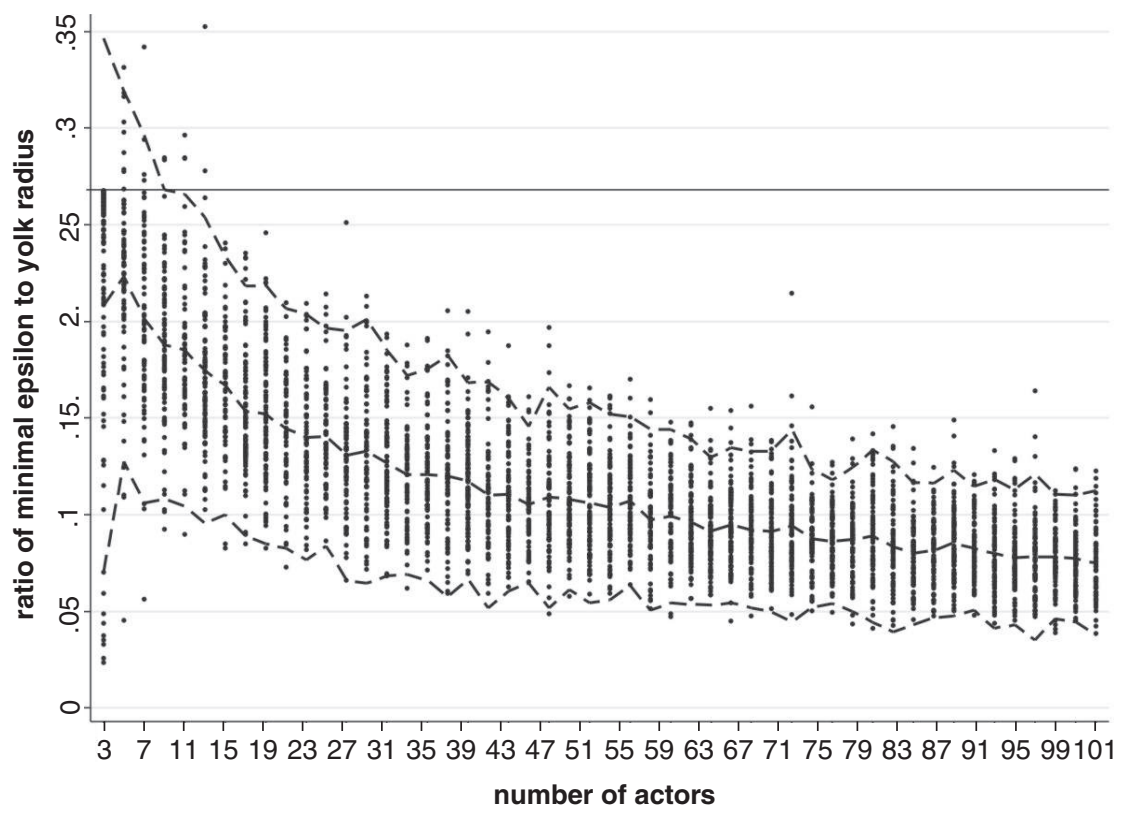

Note. The solid line is the average ratio, dashed lines are upper and lower bound ratios (mean \pm 2 standard deviations).

Figure 7. Ratio of Minimal Threshold to Yolk Radius by Committee Size

\section{Conclusion}

What does that all mean for the analysis of committee voting? Essentially, there are two scenarios. First, if the distribution of voters' ideal points is somehow symmetric, then a median in all directions or a stable outcome will exist in the conventional spatial model. In this case, $\varepsilon^{*}$ equals zero and the median in all directions, the yolk center, the finagle point and the $\varepsilon$-core coincide. Second, and from a generic standpoint, if the voter distribution deviates from symmetry, a multi-dimensional median will not exist, and the yolk center will generally be different from the finagle point. In this case, and if the act of voting is not costless for the committee members but comes with some decision costs, the finagle point rather than the center of the yolk represents the best approximation of likely outcomes. The finding is in line with theoretical, experimental and simulation analyses which show that stable outcomes under restricted voting can be found 'near the center of the preference space' (Koehler, 2001: 166). The above analysis suggests that this center of the preference space is the finagle point rather than the yolk center. Finally, the minimal voting threshold that guarantees stable outcomes is, even for modest committee sizes, small and decreases as the 
committee size increases. This suggests that given the strict and probably unreasonable assumptions of the conventional spatial model as regards the costlessness of voting, stable, preference- and not only institution-induced outcomes can indeed be expected in real world politics.

\section{REFERENCES}

Arrow, Kenneth J. (1951) Social Choice and Individual Values. New York: Wiley.

Black, Duncan (1958) The Theory of Committees and Elections. Cambridge: Cambridge University Press.

Bräuninger, Thomas (2003) 'When Simple Voting Doesn't Work: Multicameral Systems for the

Representation and Aggregation of Interests in International Organizations', British Journal of Political Science 33: 681-703.

Davis, Otto, Morris DeGroot and Melvin Hinich (1972) 'Social Preference Orderings and Majority

Rule', Econometrica 40: 147-57.

Downs, Anthony (1957) An Economic Theory of Democracy. New York: Harper.

Eavey, Cheryl and Gary Miller (1984) 'Fairness in Majority Rule Games with a Core', American Journal of Political Science 28: 570-86.

Enelow, James M. and Melvin J. Hinich (1984) The Spatial Theory of Voting: An Introduction. Cambridge: Cambridge University Press.

Feld, Scott L. and Bernard Grofman (1996) 'Stability Induced by "No-Quibbling”', Group Decision and Negotiation 5: 283-94.

Ferejohn, John A., Richard D. McKelvey and Edward W. Packel (1984) 'Limiting Distributions for Continuous State Markov Voting Models', Social Choice and Welfare 1: 45-67.

Fiorina, Morris and Charles R. Plott (1978) 'Committee Decisions under Majority Rule', American Political Science Review 72: 575-98.

Ganghof, Steffen and Thomas Bräuninger (2006) 'Government Status and Legislative Behaviour. Partisan Veto Players in Australia, Denmark, Finland and Germany', Party Politics 12: 521-39.

Godfrey, Joseph and Nicholas Miller (2005) 'On the Size and Location of the Yolk: Results Using CyberSenate Software'. Paper presented at the Public Choice Society Meeting, New Orleans, Lousiana, 10-13 March.

Greenberg, Joseph (1979) 'Consistent Majority Rules over Compact Sets of Alternatives', Econometrica 47: 627-36.

Hammond, Thomas H. and Gary J. Miller (1987) 'The Core of the Constitution', American Political Science Review 81: 1155-74.

Herzberg, Roberta and Rick Wilson (1991) 'Costly Agendas and Spatial Voting Games: Theory and Experiments on Agenda Access Costs', in Thomas Palfrey (ed.) Experimentation in Political Science, pp. 169-99. Ann Arbor, MI: University of Michigan Press.

Hinich, Melvin J. and Michael C. Munger (1997) Analytical Politics. Cambridge: Cambridge University Press.

Hotelling, Harold (1929) 'Stability in Competition', Economic Journal 39: 41-57.

Hug, Simon (1999) 'Nonunitary Agents in Spatial Models', Journal of Conflict Resolution 43: 479-500.

Koehler, David H. (1990) 'The Size of the Yolk: Computations of Odd and Even-numbered Committees', Social Choice and Welfare 7: 231-45.

Koehler, David H. (1996) 'Committee Choice and the Core under Supramajority Rule: Results from Simulation of Majority Choice in Two-dimensional Space', Public Choice 87: 281-301.

Koehler, David H. (2001) 'Convergence and Restricted Preference Maximizing under Simple Majority Rule: Results from a Computer Simulation of Committee Choice in Two-Dimensional Space', American Political Science Review 95: 155-67.

Kovalenkov, Alexander and Myrna H. Wooders (2000) 'Epsilon Cores of Games with Limited Side Payments: Nonemptiness and Equal Treatment', Games and Economic Behavior 36: 193-218. 
Laver, Michael (2005) 'Policy and the Dynamics of Political Competition', American Political Science Review 99: 263-81.

McKelvey, Richard D. (1976) 'Intransitivities in Multidimensional Voting Models and Some Implications for Agenda Control', Journal of Economic Theory 12: 472-82.

Maschler, Michael, Bezalel Peleg and Lloyd S. Shapley (1979) 'Geometric Properties of the Kernel, Nucleolus, and Related Solution Concepts', Mathematics of Operations Research 4: 303-38.

Miller, Nicholas R., Bernard Grofman and Scott L. Feld (1989) 'The Geometry of Majority Rule', Journal of Theoretical Politics 1: 379-406.

Nakayama, Akira and Takeshi Naitoh (1999) 'On some Properties of the $\varepsilon$-core of Games with Coalition Structure', International Journal of Game Theory 28: 253-5.

Plott, Charles R. (1967) 'A Notion of Equilibrium and its Possibility Under Majority Rule', American Economic Review 57: 787-806.

Poole, Keith T. and Howard Rosenthal (1997) Congress: A Political-economic History of Roll Call Voting. New York: Oxford University Press.

Riker, William H. and Peter Ordeshook (1968) 'A Theory of the Calculus of Voting', American Political Science Review 62: 25-42.

Salant, Stephen W. and Eban Goodstein (1990) 'Predicting Committee Behavior in Majority Rule Voting Experiments', RAND Journal of Economics 21: 293-313.

Samuelson, Larry (1984) 'Electoral Equilibria with Restricted Strategies', Public Choice 43: 307-27.

Schofield, Norman J. (1978) 'Instability of Simple Dynamic Games', Review of Economic Studies 45: 575-94.

Shapley, Lloyd S. and Martin Shubik (1966) 'Quasi-cores in a Monetary Economy and Nonconvex Preferences', Econometrica 34: 805-28.

Shepsle, Kenneth A. (1979) 'Institutional Arrangements and Equilibrium in Multidimensional Voting Models', American Journal of Political Science 23: 27-69.

Shepsle, Kenneth A. and Barry R. Weingast (1981) 'Structure Induced Equilibrium and Legislative Choice', Public Choice 37: 503-19.

Sloss, Judith (1973) 'Stable Outcomes in Majority Voting Games', Public Choice 15: 19-48.

Tovey, Craig (1991) The Instability of Instability. Technical Report NPSOR, Vol. 91/15. Monterey, CA: Department of Operations Research, Naval Postgraduate School.

Tovey, Craig (1992) The Almost Surely Shrinking Yolk. Working Paper, Vol. 161. Washington University Center in Political Economy.

Tsebelis, George (2002) Veto Players: How Political Institutions Work. Princeton, NJ: Princeton University Press.

Tullock, Gordon (1981) 'Why So Much Stability?', Public Choice 37: 189-202.

Wuffle, A., Scott L. Feld, Guillermo Owen and Bernard Grofman (1989) 'Finagle's Law and the Finagle Point: A New Solution Concept for Two-candidate Competition in Spatial Voting Games Without a Core', American Journal of Political Science 33: 348-75.

THOMAS BRÄUNINGER is Emmy Noether Fellow and Junior Fellow at the ZWN, University of Konstanz. His research interests are in comparative politics, political economy and formal political theory. His articles have appeared in the British Journal of Political Science, European Journal of Political Research, Journal of Conflict Resolution, Journal of Theoretical Politics, Political Studies, Public Choice, and Party Politics, among others. ADDRESS: Department of Politics and Management, University of Konstanz, 78457 Konstanz, Germany [email: thomas.braeuninger@uni-konstanz.de]. 